\title{
DESIGN OF COST EFFECTIVE PROSTHETIC ARM FOR DISABLED
}

\author{
Shridhar Anant Walavalkar ${ }^{1}$ and Pavankumar R. Sonawane ${ }^{2}$
}

\begin{abstract}
The aim of this work is to design prosthetic robotic arm for disabled using cost effective proposal to perform different object picking tasks. The robotic arm consists of a wrist and five-finger gripper. It can perform different gripping actions, such as spherical, cylindrical and tip-holding gripping actions using a five-finger gripper; each finger has three movable links. The actuator used for the robotic arm is a servo motor, and the five-finger gripper consists of five cables placed like tendons in the human arm. We have created two proposals for prosthetic arm \& FEA used for validation. CAD modeling of prosthetic robotic arm has done using Catia V5 R19. FEA analysis using ANSYS 15.0 has been carried out to fond optimum shape of finger geometry.
\end{abstract}

Keywords - Prosthetic Arm, Cost Effective, Disabled, Design, FEA analysis

\section{INTRODUCTION}

Human hand is having very high capacities \& capabilities. Arm is very important part of the body \& can't be replaced naturally. Prosthetic limbs are commonly used by people with limb loss (acquired amputation) and limb absence (congenital deficiency) to restore some of the functions and/or cosmetics purpose. If upper limb prosthesis could truly replace the human hand and arm, the job of healthcare professionals would be easy. Unfortunately, prosthesis can perform only a few of the functional motions of our arms and hands.

Various efforts has been carried out on design of prosthetic arm but available solutions are costly hence cannot affordable for peoples from developing countries like India. Use of robotic parts will help in quick, precise \& smooth movements of fingers. Our aim is to come up with cost effective solution for disabled with competitive functions. Which will help disabled person in daily life day to day activities \& will make them independent from others help.

\section{METHODOLOGY}

Following are important phases of methodology

${ }^{1}$ Department of Mechanical Engineering D. Y. Patil Institute of Engineering \& Technology, Ambi, Pune, Maharashtra, India

${ }^{2}$ Department of Mechanical Engineering D. Y. Patil Institute of Engineering \& Technology, Ambi, Pune, Maharashtra, India 


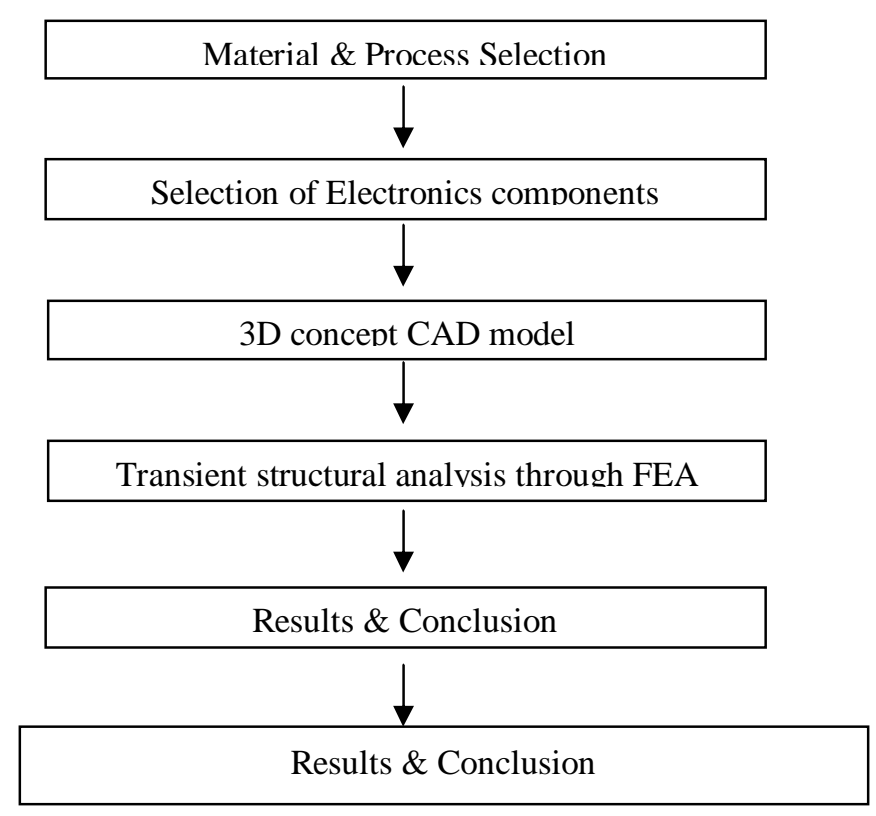

Figure 1. Methodology Block Diagram

\section{A. Material \& Process Selection}

Various alternative material options are available in market. Light weight metals, Alloys, Plastics etc. are available options for this project. Selection factors are density, Ultimate Tensile Strength, Manufacturing process and total manufacturing cost. In metals we have selected Aluminium alloy 6061-T6 which is widely used for Aluminium cans for the packaging of food and beverages, automotive parts such as wheel spacers, construction of aircraft structures, such as wings and fuselages etc.

Whereas ABS (Acrylonitrile butadiene Styrene) is selected from plastics because of availability of $3 \mathrm{D}$ printing process is low cost manufacturing process. ABS's light weight and ability to be injection molded and extruded make it useful in manufacturing products such as drain-wastevent (DWV) pipe systems, musical instruments (recorders, plastic clarinets, and piano movements), golf club heads (because of its good shock absorbance), automotive trim components, automotive bumper bars, medical devices for blood access, enclosures for electrical and electronic assemblies, Household and consumer goods are the major applications of ABS.

ABS material is selected because of availability of low cost manufacturing process - 3D printing compared with other materials, Light weight $\left(\right.$ Density $=1100 \mathrm{Kg} / \mathrm{m}^{3}$ ) and having better load carrying capacity.

\section{B. Selection of Electronics components}

a. Microcontroller-

A microcontroller is a computing device capable of executing a program (i.e. a sequence of instructions) and is often referred to as the "brain" or "control center" in a robot since it is usually responsible for all computations, decision making, and communications. Following factors are considered while selection of microcontroller:

1) Popularity in robotics world 
There are various benefits of using popular microcontroller because it is common for robot builders to share results, code, pictures, videos, and detail successes and even failures. All this available material and the possibility of receiving advice from more experienced users can prove very valuable.

2) Inputs to microcontroller

Apart from basic common features of microcontrollers while others need specific hardware (e.g ADC, PWM, and communication protocol support). Also memory and speed requirements, as well as pin count taken into consideration.

3) Compatibility of my accessories with microcontroller

Although most sensors and accessories can be interfaced directly with many microcontrollers, some accessories are meant to interface with a specific microcontroller and even provide out-ofthe-box functionally or sample code.

4) Cost

The microcontroller's cost must be low s possible which will results in effective low cost solution. 5) Specifications

Since our robot will use five motors, the microcontroller will need five digital pins for direction control, and five PWM pins for speed control.

Based on above parameters we have selected Arduino Uno R3 with cable. The Arduino Uno is a microcontroller board based on the ATmega328 (datasheet). It has 14 digital input/output pins (of which 6 can be used as PWM outputs), 6 analog inputs, a $16 \mathrm{MHz}$ ceramic resonator, a USB connection, a power jack, an ICSP header, and a reset button. It contains everything needed to support the microcontroller; simply connect it to a computer with a USB cable or power it with an AC-to-DC adapter or battery to get started. The Arduino Uno can be powered via the USB connection or with an external power supply. The power source is selected automatically. The Arduino Uno has a number of facilities for communicating with a computer, another Arduino, or other microcontrollers. The Arduino Uno can be programmed with the Arduino software. The ATmega328 on the Arduino Uno comes preburned with a bootloader that allows user to upload new code to it without the use of an external hardware programmer. It communicates using the original STK500 protocol.

\section{b. Actuator.}

Various rotational actuators are available in market such as AC Motor, DC Motor, Geared DC motors, R/C servo motor, Stepper motor etc. Selection factors are:

\section{1) Range of motion}

If the range is limited to 180 degrees and the torque required is not significant, an R/C servo motor is ideal. Servo motors are offered in a variety of different torques and sizes and provide angular position feedback (most use a potentiometer, and some specialized ones use optical encoders). R/C servos are used more and more to create small walking robots.

2) Specifications

Here we have considered finger as a rigid cantilever beam. Four fingers need to hold $1 \mathrm{Kg}$ of load. So free body diagram will be as shown below cantilever beam fixed at one end \& load of $1.2 \mathrm{Kg}$ at other end. 


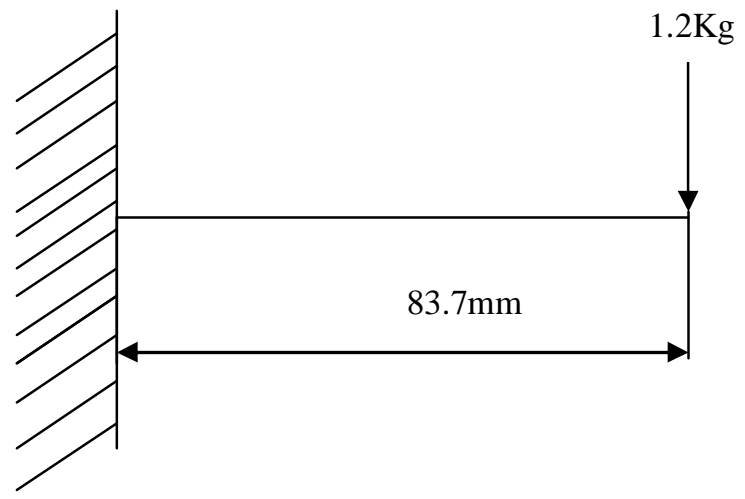

Figure 2. Free Body diagram finger load

Therefore moment at fixed beam will be $=1.2 \times 83.7=100.44 \mathrm{Kgmm}=10.5 \mathrm{Kgcm}$.So our selected servo motor is Towerpro Mg995 metal gear Servo Motor is having following specifications:

\begin{tabular}{|l|l|}
\hline Operating Speed & $\begin{array}{l}0.17 \mathrm{sec} / 60 \text { degrees (4.8V no load), } \\
0.13 \mathrm{sec} / 60 \text { degrees }(6.0 \mathrm{~V} \text { no load })\end{array}$ \\
\hline Stall Torque & $\begin{array}{l}9.2 \mathrm{~kg}-\mathrm{cm}(180.5 \mathrm{oz}-\mathrm{in}) \text { at } 4.8 \mathrm{~V}, \\
12 \mathrm{~kg}-\mathrm{cm}(208.3 \mathrm{oz}-\mathrm{in}) \text { at } 6 \mathrm{~V}\end{array}$ \\
\hline Operation Voltage & $4.8-7.2$ Volts \\
\hline Gear Type & All Metal Gears \\
\hline Bearings & Dual \\
\hline Cost approx. (INR) & 490 \\
\hline
\end{tabular}

Figure 3. Towerpro Mg995 metal gear Servo Motor specifications

\section{DESIGN OF PROSTHETIC ARM}

By using Computer Aided Design 3D model is generated. Catia V5 R19 software had used for modeling, drafting \& Simulation purpose. By using digital mock up workbench functionality has checked \& modifications in model created as per requirements. Two proposals created, one with circular cross section $\&$ other with rectangular.

3D CAD model has created using Catia V5 R19. Proposal one is having circular cross section whereas proposal two is having square cross section. Overall dimensions such as total length, width of finger, length of finger etc. are kept same in both proposals. Figure below shows overall dimensions of proposal -1 . Proposal -2 is having same overall dimensions. Also exploded views, simulation is shown only for proposal -1 . 


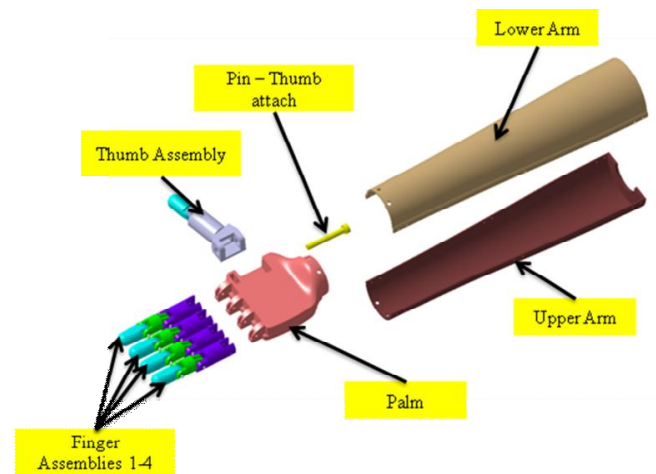

(a)

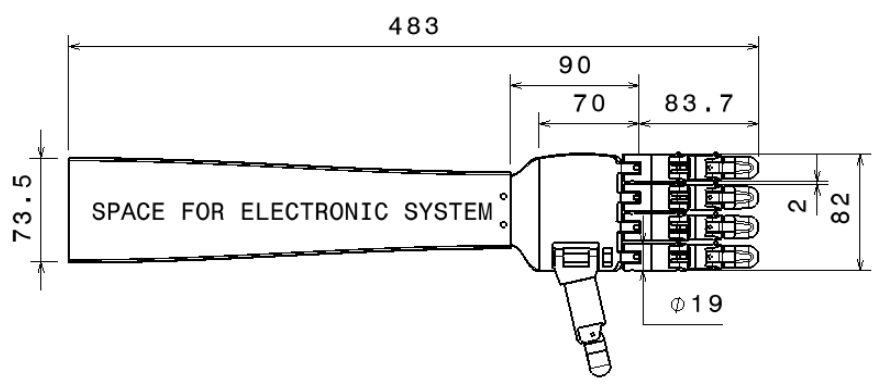

SCALE: $\quad 1: 4$

(b)

Figure 4. (a)Model Exploded view, (b) Overall Dimensions (Proposal - 1)

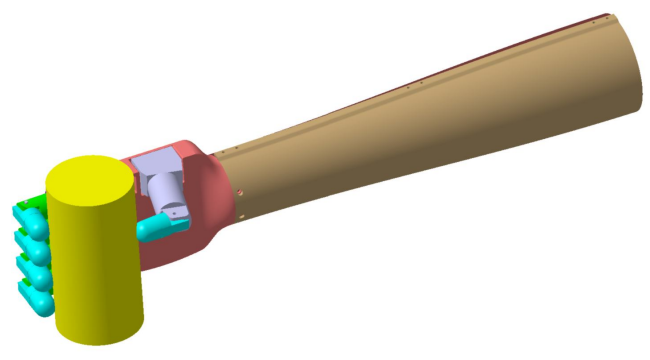

(a)

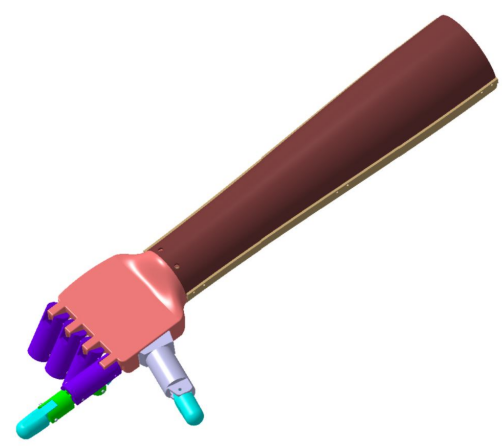

(b)

Figure 5. (a)Simulation of $\phi 60 \mathrm{~mm}$ object holding, (b) Simulation of Typing

\section{EXPERIMENT AND RESULT}

Finite element Analysis carried out by using ANSYS 15.0. As fingers will in direct contact with object to hold, only analysis of fingers is carried out. Transient Structural analysis is used. Step by step procedure is explained below.

1. Material properties

As explained earlier ABS is selected as a material \& following properties were considered for Finite Element Analysis.

Density $(\mathrm{Kg} / \mathrm{m} 3)=1100$,

Ultimate Tensile Strength $(\mathrm{MPa})=40$,

Modulus of Elasticity $(\mathrm{MPa})=2300$,

Poisson's Ratio $=0.4$,

Yield Tensile Strength $(\mathrm{MPa})=25$.

2. Meshing

Fine size meshing is used for accurate results. 


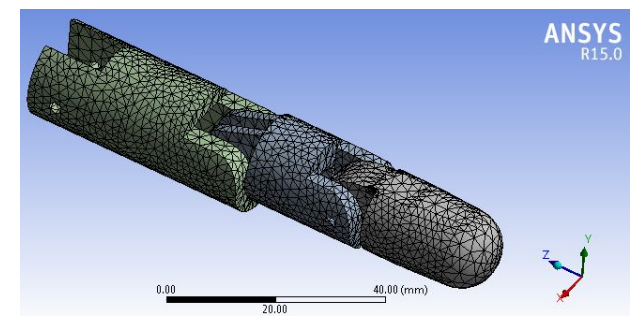

Figure 6. Meshing of Proposal $-1($ Nodes $=33827$, Elements $=19030)$

\section{Boundary Conditions}

Revolute joints have been applied at all three joints. Rotation angles used are Joint A$=60^{\circ}$, Joint $\mathrm{B}=64.5^{\circ} \&$ Joint $\mathrm{C}=81^{\circ}$.

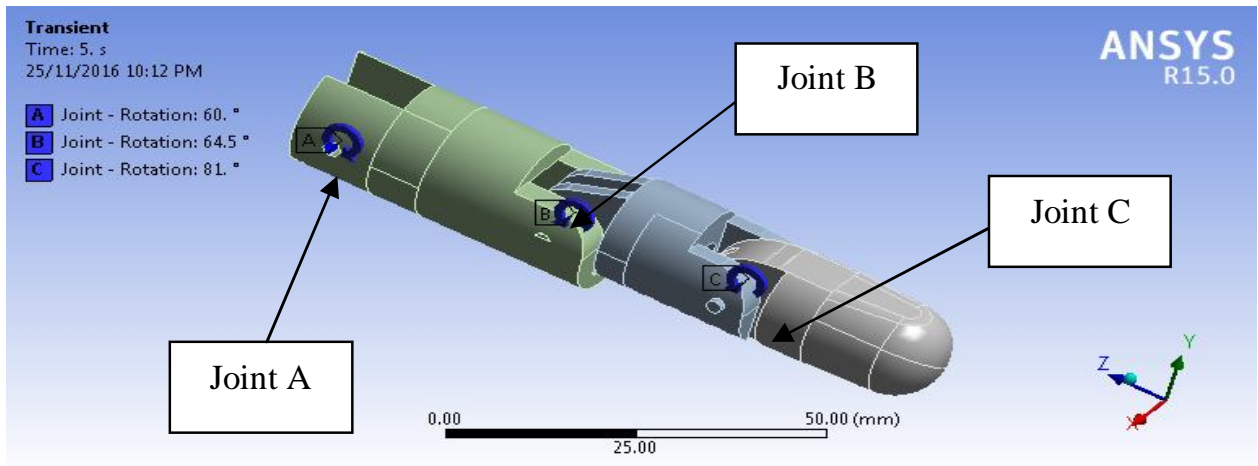

Figure 7. Boundary conditions (Proposal -1)

Figure 8.

4. Results

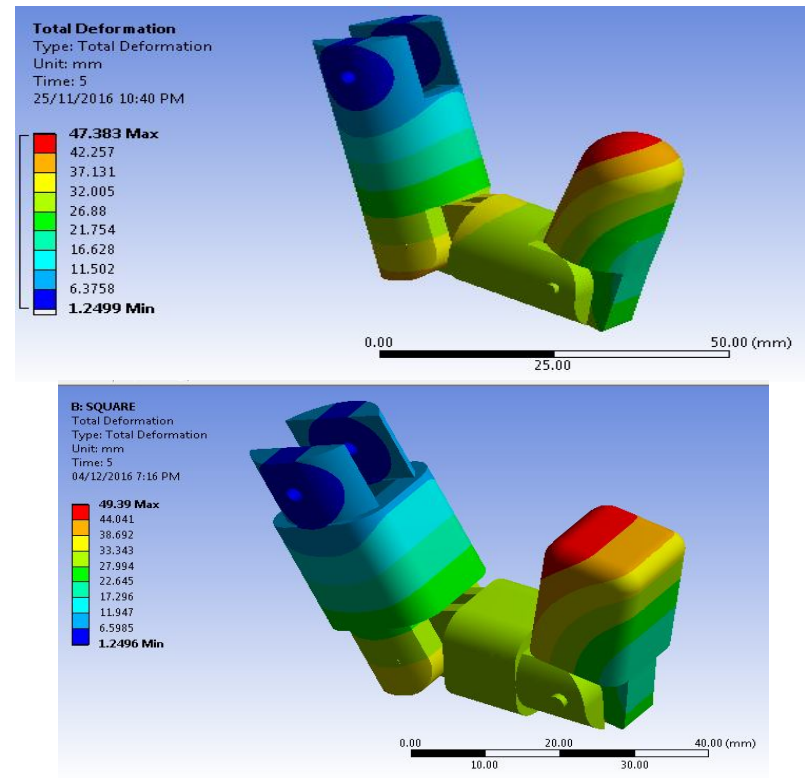

(a)

(b)

Figure 9. (a) Total Deformation (Proposal - 1), (b) Total Deformation (Proposal - 2) 

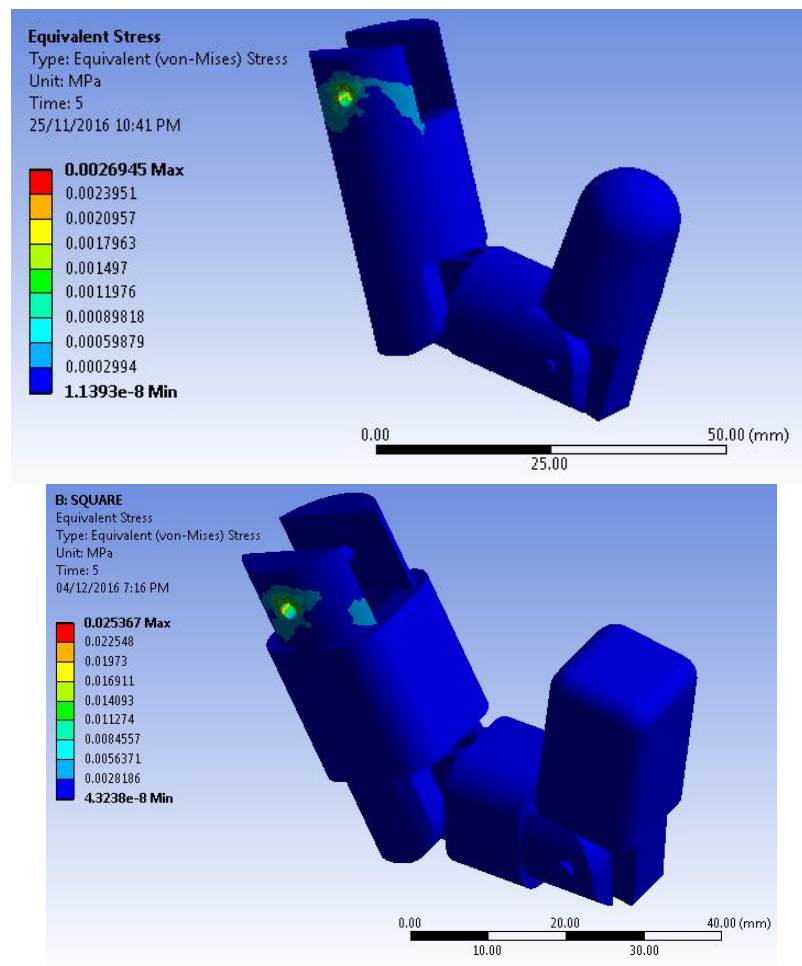

(a)

(b)

Figure 10. (a) Equivalent Stress (Proposal - 1), (b) Equivalent Stress (Proposal - 2)

Table -1 Experiment Result

\begin{tabular}{|l|l|l|l|}
\hline $\begin{array}{l}\text { Sr. } \\
\text { No. }\end{array}$ & Results & $\begin{array}{l}\text { Proposal } \\
(\text { Round c/s) }\end{array}$ & $\begin{array}{l}\text { Proposal -2 (Square } \\
\text { c/s) }\end{array}$ \\
\hline 1 & $\begin{array}{l}\text { Total Deformation } \\
\text { max (mm) }\end{array}$ & 47.383 & 49.39 \\
\hline 2 & $\begin{array}{l}\text { Equivalent Stress } \\
\text { max (Mpa) }\end{array}$ & 0.015362 & 0.025367 \\
\hline
\end{tabular}

\section{V.CONCLUSION}

Results obtained from Ansys it shows that both proposals can be safe to perform its motion. Also there is negligible difference in mass of both the proposals \& in their FEA results. But proposal -1 is modular \& simple than proposal-2. Therefore proposal -1 cross section area circular is selected for next phase.

\section{REFERENCES}

[1] Sharma A., Noel M., "Design of a low-cost five-finger anthropomorphic robotic arm with nine degrees of freedom”, Elsevier, pp.551-558,2012. 
[2] Kumar V.S., Aswath S., Shashidhar T. and Choudhary R., "A Novel Design of a Full Length Prosthetic Robotic Arm for the Disabled”, Springer, pp.271-287, 2017.

[3] Elfasakhany, A, Yanez E., Baylon K., Salgado, R, "Design and development of a competitive low-cost robot arm with four degrees of freedom", Scientific Research. pp. 47-55, 2011.

[4] Vijayan A., Nutakki C., Medini C., Singanamala H., Nair B.., "Classifying Movement Articulation for Robotic Arms via Machine Learning", Journal of Intelligent Computing, pp.123-134, 2013.

[5] Verma S. \& Chatterjee A. \& Singh H. \& Kumar A. \& Garg K. \& Chattopadhyay K., "A design approach for myoelectric arm with hand and wrist motions using single actuator", Springer, pp.1899-1907, 2012.

[6] Yang D., Jiang L., Huang Q, Liu R., Liu H., "Experimental study of an EMG-Controlled 5-DOF Anthropomorphic Prosthetic Hand for Motion Restoration", Springer,2014

[7] Saleh A., Negm F., Said N., Sudan Y., Halawa H., Daoud R., Amer H., "Wireless Body Area Network Architecture for a Prosthetic Arm”, IEEE, pp. 275-280, 2015.

[8] Martin H., Donaw J., Kelly R., Jung Y., Kim J., "A Novel Approach of Prosthetic Arm Control using Computer Vision, Biosignals, and Motion Capture", IEEE, 2014.

[9] Ciarán O'Neill, an “Advanced, Low Cost Prosthetic Arm”, IEEE, 2014.

[10] Bandara D., Gopura C., "A multi-DoF Anthropomorphic Transradial Prosthetic Arm”, pp.1039-1044, IEEE, 2014.

[11] Patrik K., Jan H., Jan K. and Slavka V., "Prosthetic 6-DOF Arm Controlled by EMG Signals and MultiSensor System", IEEE, 2013.

[12] Neogi B., Ghosal S., Ghosh S., Bose T., Das A., "Dynamic Modeling and Optimizations of Mechanical Prosthetic Arm by Simulation Technique", IEEE, 2012.

[13] Takeda H., Tsujiuchi N., Koizumi T., Kan H., "Development of Prosthetic Arm with Pneumatic Prosthetic Hand and Tendon-Driven Wrist", IEEE, pp. 5048-5051, 2009.

[14] Fei Gaoa, Hua Denga, Yi Zhanga, "Hybrid Actuator Combining Shape Memory Alloy with DC Motor for Prosthetic Fingers", Elsevier, pp. 1-17, 2014.

[15] Belter J., Segil J., Dollar A., "Mechanical design and performance specifications of anthropomorphic prosthetic hands: A review", JRRD, pp. 599-617, 2013. 